The influence of different surface terminations on electrical transport and emission properties for freestanding single crystalline (100) CVD diamond samples

Non Peer-reviewed author version

DEFERME, Wim; BOGDAN, Anna; HAENEN, Ken; DE CEUNINCK, Ward; Flipse, K. \& NESLADEK, Milos (2008) The influence of different surface terminations on electrical transport and emission properties for freestanding single crystalline (100) CVD diamond samples. In: Nebel, CE \& Jackman, RB \& Nemanich, RJ \& Nesladek, $M$ (Ed.) MATERIALS RESEARCH SOCIETY SYMPOSIUM PROCEEDINGS. p. 83-89..

Handle: http://hdl.handle.net/1942/8447 


\section{The Influence of Different Surface Terminations on Electrical Transport and Emission Properties for Freestanding Single Crystalline (100) CVD Diamond Samples}

Wim Deferme ${ }^{1}$, Andrey Bogdan ${ }^{1}$, Ken Haenen ${ }^{1,2}$, Ward De Ceuninck ${ }^{1,2}$, Kees Flipse ${ }^{3}$, and Milos Nesladek $^{1,4}$

${ }^{1}$ Institute for Materials Research, Hasselt University, Wetenschapspark 1, Diepenbeek, 3590, Belgium

${ }^{2}$ Division IMOMEC, IMEC vzw, Wetenschapspark 1, Diepenbeek, 3590, Belgium

${ }^{3}$ Physics Department, Eindhoven Univ. of Technology, Eindhoven, Netherlands

${ }^{4}$ LIST (CEA-Recherche Technologique)/DETECS/SSTM/LTD, CEA/Saclay, Gif-sur-Yvette, France

\section{ABSTRACT}

The surface density of states of hydrogen and oxygen terminated diamond is an important parameter from the point of view of electrical transport properties at the surface. In addition, the presence of surface states has a detrimental influence on the electrical contact properties. Therefore it is of great importance that the influence of different species on the surface-related properties of the diamond layer is well understood.

In this work (100)-oriented CVD diamond films are terminated using a hydrogen plasma with and without small additions of oxygen (1 to 4\%). XPS and UPS measurements are performed to look at the influence of this addition on the surface band gap of the diamond samples. Using the TOF (Time-of-Flight) technique a comparison is made between oxidized and (differently) hydrogenated diamond surfaces. The results clearly show that the different terminations of the diamond surface have an influence on the electrical transport properties. For the oxidized surface, it is found that defect states are created in the surface band gap, increasing trapping. Contrary to this, fully hydrogenated layers behave differently in a way that suggests that the surface DOS is significantly reduced. This fact can be confirmed when applying an electric field on the CVD diamond samples sandwiched between two metallic electrical contacts. In case of a metal deposited on a hydrogen terminated surface one can see a clear UV light emission related to free exciton recombination in the contact-diamond-junction region. When the surface is oxidized the UV emission is damped, an effect which is attributed to the parallel recombination channels via the surface states.

\section{INTRODUCTION}

Single crystal diamond is throughout the complete array of wide band gap materials the most promising one for use in bio-electronic applications. In addition, the high thermal conductivity, the high mobility and the high electrical breakdown field make diamond an interesting material for devices such as Schottky diodes and field-effect transistors.[1-3] It is known that an hydrogen termination of the diamond surface gives rise to a high p-type surface conductivity and to a negative electron affinity.[4,5] Applications based on these peculiar properties of the diamond layer have been developed. Even opto-electronic applications based on 
the ability to emit ultraviolet (UV) light have already been fabricated.[6] With the aim to better understand the hydrogen-induced transport properties and the emission properties, different techniques are used to investigate the electrical and emission properties for differently terminated diamond surfaces.

Thin single crystal diamond (100)-oriented layers, grown on Ib HPHT diamond epilayers, are terminated under different conditions. A comparison is made between layers that are fully oxidized, fully hydrogenated or hydrogenated in a hydrogen plasma containing 1 to $4 \%$ of oxygen. X-ray Photoelectron Spectroscopy (XPS) is used to examine the different bonding configurations of oxygen at the diamond (sub)-surface. Ultraviolet Photoelectron Spectroscopy (UPS) is used to show that the samples hydrogenated in a $1 \% \mathrm{H}_{2} / \mathrm{O}_{2}$ plasma still have negative electron affinity $(-0.3 \mathrm{eV})$, however reduced in comparison with the fully hydrogenated layer ($2.2 \mathrm{eV}$ ), while the work function is $3.4 \mathrm{eV}$.

Furthermore, the Time-of-Flight (TOF) technique is utilized to investigate the influence of the different terminations on the electrical transport properties. It is shown that, for the completely oxidized diamond surface, trapping occurs induced by defect states created in the surface band gap, while for the completely hydrogenated samples, less trapping occurs. This phenomenon is also confirmed by using optical spectroscopy (OS). This technique clearly points out that for the hydrogenated diamond surfaces, a luminescence located at $\sim 235 \mathrm{~nm}$ is observed, while this luminescence peak, attributed to free excitons, is not as clearly distinguished for the oxidized surface.

\section{EXPERIMENT}

(100)-oriented single crystal layers, prepared by chemical vapour deposition (CVD) are chemically oxidized in a mixture of $\mathrm{H}_{2} \mathrm{SO}_{4}$ and $\mathrm{KNO}_{3}$ in order to remove all surface contamination. The termination of the layers is done in a hydrogen plasma at $700^{\circ} \mathrm{C}$ with an addition off 0-4\% of oxygen. XPS and UPS measurements are performed using a HP5950A with an energy resolution of $0.6 \mathrm{eV}$ with the $\mathrm{Al} \mathrm{K} \mathrm{K}_{\alpha}$ line $(h v=1486.6 \mathrm{eV})$ as the source for the XPS measurements. The UPS measurements are carried out to determine the electron affinity $(\chi)$ and work function $(\phi)$ of the different samples.

For the TOF measurements, the terminated layers are covered with aluminum contacts on both sides of the diamond layer so that the device will behave as a double reversed Schottky diode structure. The top contact $(20 \mathrm{~nm} \mathrm{Al}$ ) has a transparency of $\sim 30 \%$ at $220 \mathrm{~nm}$ and a resistance of less than $1 \mathrm{k} \Omega$, while the back contact is non-transparent at $220 \mathrm{~nm}$ and is $50 \mathrm{~nm}$ thick. For the hydrogenated layer, after contact deposition, the H-terminated layer around the Al contact is removed using an oxygen plasma, so the H-termination only remains under the top contact.

The TOF technique was used to measure the mobility of the diamond samples. A $2.7 \mathrm{~ns}$ pulse is created using a Nd:YAG pumped optical paramagnetic oscillator (OPO), tuneable from 420 till $700 \mathrm{~nm}$ and doubled by BBO crystals. A wavelength of 215-220nm is generated, which is just above the band gap energy of intrinsic diamond. The main voltage pulse (4ms length) is 100x amplified and applied to the sample to assure a homogeneous electric field. The sheet of carriers, created in the first $10 \mu \mathrm{m}$ of the diamond layer, travels through the diamond from the top contact to the bottom contact under an electric field varied from $0.3 \mathrm{~V} / \mu \mathrm{m}$ to $1.4 \mathrm{~V} / \mu \mathrm{m}$. The measurements were recorded using a $500 \mathrm{MHz}$ digital oscilloscope and an averaging was done 
over 25 measurements to reduce the noise according to the signal. The optical response was measured using an optical spectroscope with a cooled CCD camera.

\section{DISCUSSION}

\section{$\underline{\text { XPS and UPS measurements }}$}

In table 1 results for the XPS measurements, performed on the differently terminated samples, are shown. It can be found that for the sample hydrogenated in a $\mathrm{H}_{2} / \mathrm{O}_{2}$ plasma the surface oxygen coverage is larger than for the fully hydrogenated layer. The oxygen present at the surface of the hydrogenated layer is ascribed to exposure to air, while for the sample terminated in a $\mathrm{H}_{2} / \mathrm{O}_{2}$ plasma, part of the oxygen is coming from the process of hydrogenation, increasing the amount of $\mathrm{C}=\mathrm{O}$ bonds at the surface. The chemically oxidized sample is given as a reference.

Table 1: XPS results for samples treated in a $\mathrm{H}_{2} / \mathrm{O}_{2}$ plasma and a pure $\mathrm{H}_{2}$-plasma. A chemically oxidized sample is given as a reference.

\begin{tabular}{|c|c|c|c|c|c|c|}
\hline & & & C-peak & & & \\
\hline & $\% \mathrm{C}$ & $\% 0$ & $\mathrm{C}-\mathrm{H} / \mathrm{C}-\mathrm{C}(\%)$ & $\mathrm{C}-\mathrm{O}-\mathrm{H} / \mathrm{C}-\mathrm{O}-\mathrm{C}(\%)$ & $\mathrm{C}=\mathrm{O}(\%)$ & OTHER (\%) \\
\hline $\mathrm{H}_{2}$ & 96 & 4 & 67 & 4 & 0,5 & 28,5 \\
\hline $\mathrm{H}_{2} / \mathrm{O}_{2}$ & 93 & 7 & 69 & 2,5 & 3 & 24,5 \\
\hline \multirow{6}{*}{ Uxidized } & 88 & 12 & 45 & 12 & 4 & 39 \\
\hline & & & O-peak & & & \\
\hline & & & $\mathrm{C}=\mathrm{O}(\%)$ & $\mathrm{C}-\mathrm{O}-\mathrm{H} / \mathrm{C}-\mathrm{O}-\mathrm{C}(\%)$ & $\overline{\mathrm{C}-\mathrm{O}-\mathrm{C}=\mathrm{O}(\%)}$ & $\overline{\text { OTHER (\%) }}$ \\
\hline & & & 44,5 & $22, \overline{5}$ & 33 & 0 \\
\hline & & & 48 & 9,5 & 42,5 & 0 \\
\hline & & & 65 & 12 & 14,5 & 8,5 \\
\hline
\end{tabular}

The UPS measurements are performed to check the work function and the electron affinity of the $\mathrm{H}_{2} / \mathrm{O}_{2}$ terminated diamond layer. According to fig.1 and using the following formula:

$\chi=h v-E_{g}-\omega$

where hv is the photon energy, $\mathrm{E}_{\mathrm{g}}$ is the band gap of the diamond layer and $\omega$ is the emission width, which in this case is $35.6 \mathrm{eV}$, the electron affinity $\chi$ can be calculated. Furthermore, the electron affinity can also be calculated using the combination of the He I and the He II spectrum as can be seen in fig.1. In the He I spectrum ( $\mathrm{He} \mathrm{I}, \mathrm{hv}=21.2 \mathrm{eV}$ ), the sharp peak at low kinetic energies reveals the negative electron affinity. For the diamond layer hydrogenated in an $\mathrm{H}_{2} / \mathrm{O}_{2}$ plasma, the electron affinity is $-0.3 \mathrm{eV}$, calculated from the above formula. The work function is $3.4 \mathrm{eV}$, as indicated on fig.1. For the fully hydrogenated diamond layer, the electron affinity is about $-2.2 \mathrm{eV}$. For the oxidized sample the electron affinity is positive, stating that electrons 
excited in the conduction band can not escape into the vacuum, like is the case for the negative electron affinity from the partly hydrogenated layers. [7,8]
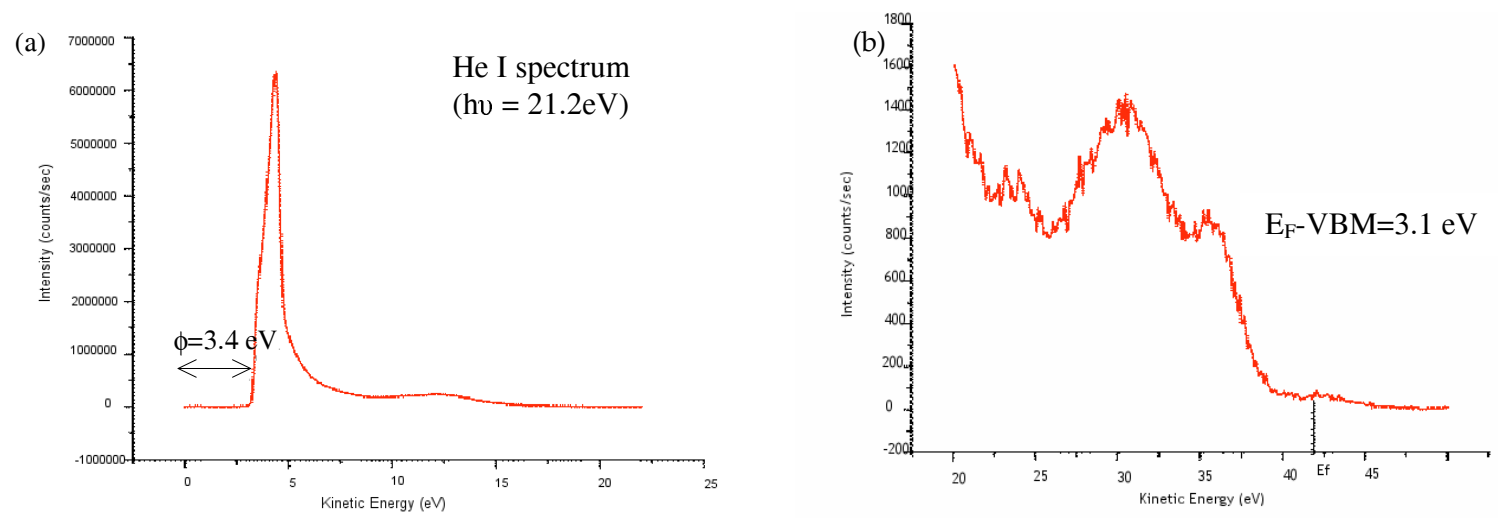

Figure 1. The UPS spectra for the sample hydrogenated in an $\mathrm{H}_{2} / \mathrm{O}_{2}$ plasma. The He I spectrum showing a peak corresponding to the NEA of the surface (a) while the He II spectrum is used to determine the Fermi level and the work function of this layer (b).

\section{TOF measurements}

TOF measurements are performed on freestanding single crystal layers. Electric fields from $0.3 \mathrm{~V} / \mu \mathrm{m}$ to $1.4 \mathrm{~V} / \mu \mathrm{m}$ (positive as well as negative polarity) are applied to the diamond layers. The laser pulse creates a sheet of carriers into the first $10 \mu \mathrm{m}$ of the surface and electrons or holes travel through the diamond layer depending on the polarity of the applied electric field. The time needed for the carriers to travel from the top contact to the bottom contact is called the transit time. From this transit time, in relationship with the thickness of the diamond sample and the applied voltage, the charge mobility can be calculated, according to this formula:

$$
\mu=\frac{d}{t_{T} \cdot E}
$$

In fig.2, typical TOF spectra for holes and electrons, for the oxidized sample can be seen. When the mobility is plotted against electric fields, the mobility values for electrons and holes can be extracted by fitting the mobility spectra by the following asymptotic behaviour formula:

$$
\mu=\frac{\mu_{0}}{1+\frac{\mu_{0} E}{v_{s}}}
$$

where $\mu_{0}$ is the mobility when no electric field is applied, $\mathrm{E}$ is the electric field and $\mathrm{v}_{\mathrm{s}}$ is the saturation velocity (1.0 $10^{7}$ for electrons and $1.210^{7}$ for holes). Mobility values as high as 
$2100 \mathrm{~cm}^{2} / \mathrm{Vs}$ for electrons and $2250 \mathrm{~cm}^{2} / \mathrm{Vs}$ for holes, can be obtained based on the numerical fitting of the experimental data, as has been reported in earlier work $[9,10]$.
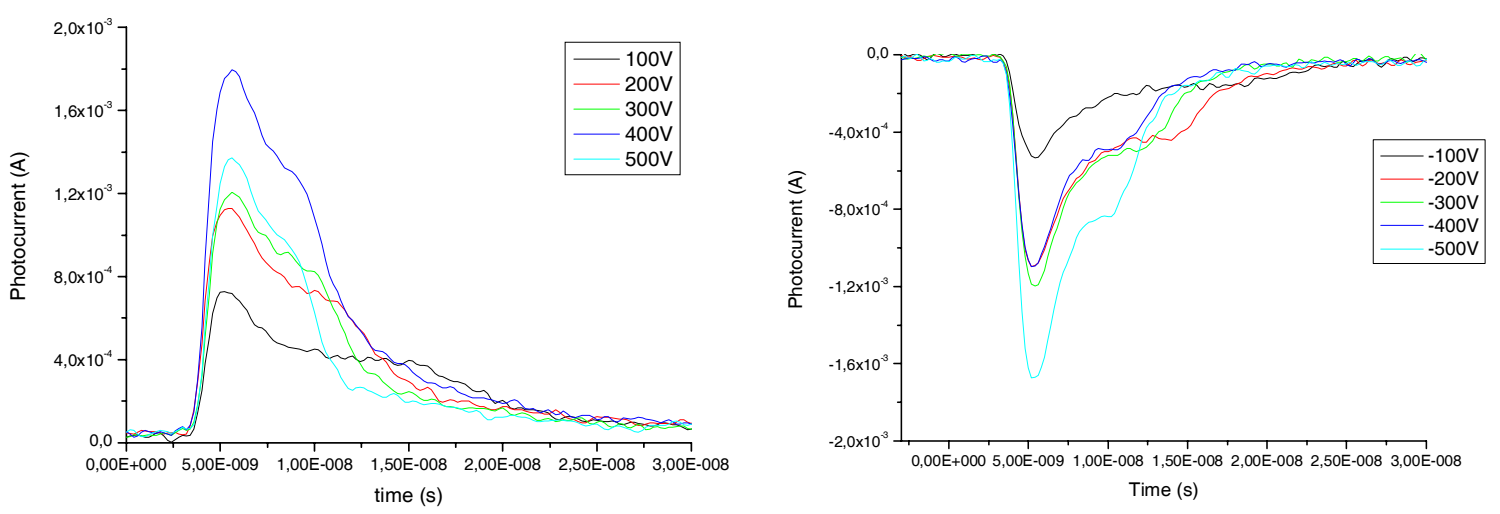

Figure 2. TOF spectra for holes (left) and electrons (right) for a surface in the oxidized state.

When measuring TOF for the hydrogenated diamond layer, a TOF spectrum like in fig.3 can be seen. It is clear that the TOF signal is very sensitive on the surface termination. For the oxidized sample the photo generated charge $Q_{o x}$ is $0.5 \times 10^{-12} \mathrm{C}$, where for the hydrogenated sample the photo generated charge $\mathrm{Q}_{\text {hydr }}$ is $1.2 \times 10^{-11} \mathrm{C}$. Comparing these values with $\mathrm{CU}$, where $\mathrm{C}$ is the sample capacity with a typical value of $0.5 \mathrm{pF}$ for $2 \mathrm{~mm}$ diameter contacts on a $500 \mu \mathrm{m}$ thick diamond layer, and $U$ is the applied voltage, one sees that for the H-terminated diamond surface has a photo generated charge larger than CU. For the oxidized sample, this value is smaller than CU. This means that the TOF measurement is in the small signal mode for the oxidized sample, where it is in the space charged limited current mode (SCLC) for the hydrogenated diamond layer. Changing the surface termination clearly switches the TOF regime from one to another. It is believed that oxygen creates defect states in the surface band gap trapping the photo generated charge shortly after these carriers have been generated by the laser flash.[11,12]

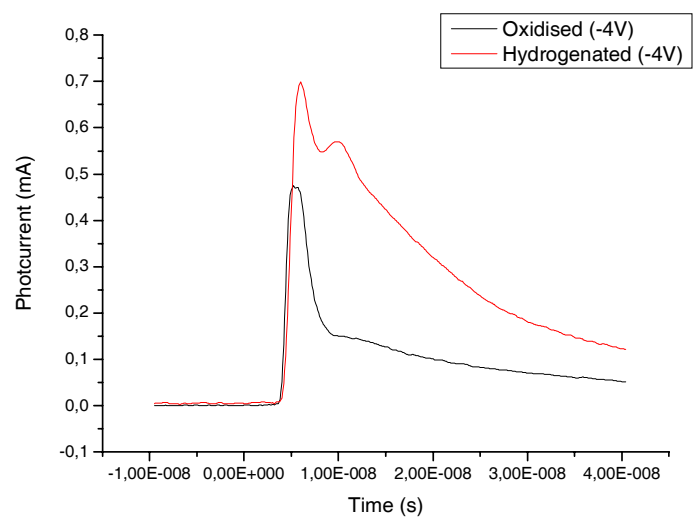

Figure 3. TOF spectrum for the hydrogenated diamond surface. The electric field used for this graph is $1.4 \mathrm{~V} / \mu \mathrm{m}$ 


\section{Optical Measurements}

In addition to these TOF measurements, optical emission is measured for the sandwiched structures. The presence of defects, like the oxygen defect in the surface band gap of the oxygen terminated diamond layers, can form recombination centers for the laser generated charge carriers. Surprisingly a visible light emission can be observed when only a voltage pulse is applied without a laser pulse. Fig.4 shows two optical spectra for the oxidized and the hydrogenated surface. For both samples a broad A-band luminescence can be seen, but in addition, a free exciton peak can be found for the hydrogenated surface. It is thought that the recombination via A-luminescent channels is reduced for the hydrogenated surface, which makes it more likely for the electron-hole pair to recombine leading to free exciton luminescence.[13] The experiments confirmed that this luminescence occurs only at high applied electric fields, and only when the polarity of the field was positive with respect to the front contact.
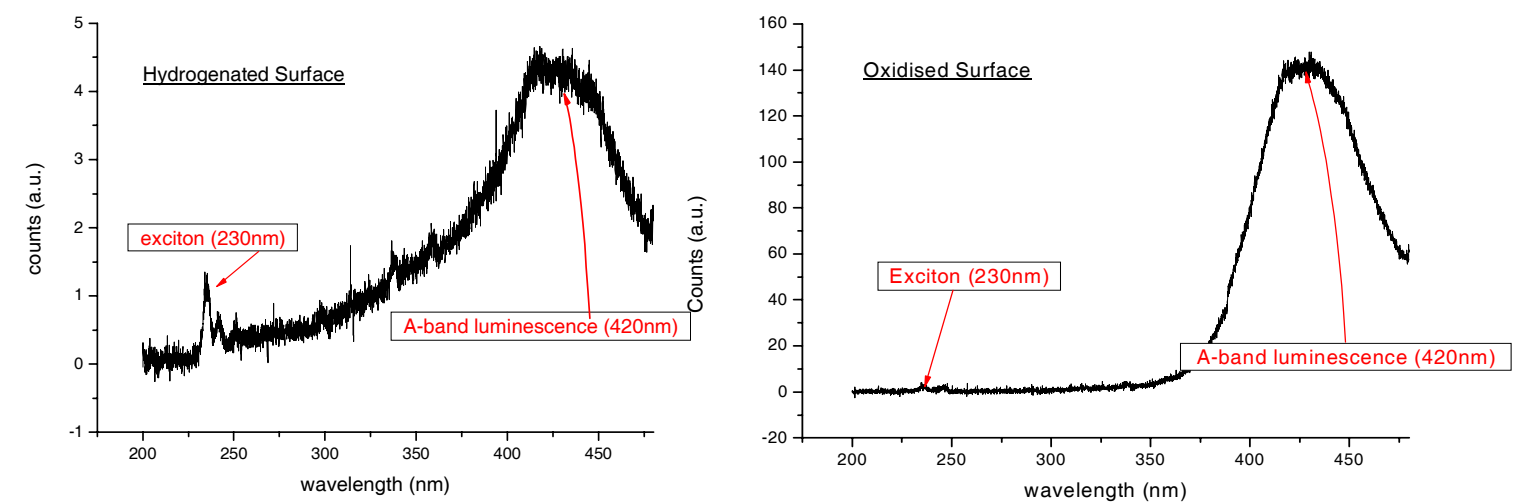

Figure 4. OS spectra for the oxidized (left) and hydrogenated (right) diamond surfaces. For the oxidized surface a broad A-band luminescence can be seen. For the hydrogenated sample, the Aband is much smaller and a free exciton peak can be found.

\section{CONCLUSIONS}

From the XPS and UPS measurements it is believed that the presence of oxygen, and the upwards band bending leads to a strong coupling of O-surface states into the valence band maximum ( $\left.\mathrm{E}_{\mathrm{VBM}}\right)$. From the TOF measurements it is clear that these O-states create defects in the surface band gap such that trapping occurs. As a result, the collected charge for the oxidized sample is smaller than for the hydrogenated one creating a switch from the small signal TOF mode to the space charge limited current mode. For the hydrogenated sample fewer defects are present and reduced charge trapping is observed. The optical emission measurements confirm these results: the hydrogenated sample shows a diminished A-band luminescence while electronhole recombination gives rise to a small free exciton peak. 


\section{ACKNOWLEDGMENTS}

This work was financially supported by the IWT-SBO-project No. 030219 "CVD Diamond: a novel multifunctional material for high temperature electronics, high power/high frequency electronics and bioelectronics", by the IAP-P5/01 project "Quantum Size Effects in Nanostructured Materials" and the EU FP6 Marie Curie Research Training Network "DRIVE", MRTN-CT-2004-512224. KH is a Postdoctoral Fellow of the Research Foundation-Flanders (FWO-Vlaanderen).

\section{REFERENCES}

1. K. Miyata, K. Nishimura, K. Kobashi, IEEE Trans. ED. 42, 2010 (1995)

2. H. Umezawa, H. Taniuchi, T. Arima, M. Tachiki, H. Kawarade, Diamond Relat. Mater. 10, $1743(2001)$

3. A. Aleksov, M. Kubovic, N. Kaeb, U. Spitzberg, A. Bergmaier, G. Dollinger, Th. Bauer, M. Schreck, B. Stritzker, E. Kohn, Diamond and Related Materials 12, 391 (2003)

4. D. Takeuchi, M. Riedel, J. Ristein, L. Ley, Phys. Rev. B 68, 041304(R) (2003)

5. J. Ristein, F. Maier, M. Riedel, J.B. Cui, L.Ley, Phys. Stat. Sol. (a) 203, 3226 (2006)

6. S. Koizumi, K. Watanabe, M. Hasegawa, H. Kanda, Science 292, 1899 (2001)

7. F. Maier, M. Riedel, B. Mantel, J. Ristein, L. Ley, Physical Review Letters 85, 3472 (2000)

8. W. Deferme, K. Haenen, G. Tanasa, C.F.J. Flipse, M. Nesladek, Phys. Stat. Sol (a) 195, 3 (2003)

9. A. Bogdan, G. Bogdan, W. De Ceuninck, K. Haenen, M. Nesladek, MRS Symp. Proc. 956, 0956-J09-22 (2007)

10. W. Deferme, A. Bogdan, G. Bogdan, K. Haenen, W. De Ceuninck, M. Nesladek, Phys. Stat. Sol (a), 204, 3017 (2007)

11. B. Rezek, C. Sauerer, C.E. Nebel, M. Stutzmann, J. Ristein, L. Ley, E. Snidero, P. Bergonzo, Appl, Phys. Lett. 82, 2266 (2003)

12. W. Deferme, G. Tanasa, J. Amir, K. Haenen, M. Nesladek, C.F.J. Flipse, Diam. Relat. Mater. 15, 687 (2006)

13. H. Okushi, H; Watanabe, S. Yamasaki, S. Kanno, Phys. Stat. Sol. (a) 203, 3226 (2006) 